\title{
Appropriate Formulations for Velocity and Pressure Calculations at Gas-liquid Interface with Collocated Variable Arrangement*
}

\author{
$\mathrm{Kei} \mathrm{ITO}^{* * * * * *}$ and Tomoaki KUNUGI ${ }^{* * *}$ \\ **Advanced Nuclear System Research and Development Directorate, Japan Atomic Energy Agency, \\ 4002 Narita, O-arai-machi, Ibaraki, 311-1393, Japan \\ E-mail: ito.kei@jaea.go.jp \\ ***Department of Nuclear Engineering, Kyoto University, \\ Yoshida-honmachi, Sakyo-ku, Kyoto-shi, Kyoto, 606-8501, Japan
}

\begin{abstract}
A high-precision simulation algorithm for gas-liquid two-phase flows on unstructured meshes has been developed to simulate gas entrainment phenomenon in a sodium-cooled fast reactor. In this study, it became clear that unphysical behaviors near gas-liquid interfaces were caused by conventional algorithms. Then, physics-basis considerations were conducted for mechanical balances at gas-liquid interfaces to derive appropriate formulations. By defining momentum and velocity independently and developing the momentum transport equations for both gas and liquid phases, the physically appropriate formulation of momentum transport was derived, which eliminated the unphysical pressure distribution caused by the conventional formulation. In addition, the physically appropriate formulation was derived for the pressure gradient to satisfy the mechanical balances between pressure and surface tension at gas-liquid interfaces. As the validation test, the rising gas bubble in liquid was simulated by the developed simulation algorithm with the physically appropriate formulations, and the simulated terminal bubble shapes on the structured and highly-distorted unstructured meshes coincided with the experimental data under each simulation condition determined by the Morton and Eötvös numbers.
\end{abstract}

Key words: Numerical Simulation, Gas-Liquid Two-Phase Flow, Free Surface Flow, Volume of Fluid, Unstructured Mesh, Gas Entrainment

\section{Introduction}

Gas-liquid two-phase flows have been of interest in various scientific and industrial fields. Owing to a number of numerical simulations and experiments performed in two-phase flow fields, a lot of knowledge has been accumulated. However, gas-liquid two-phase flows are still of interested because the existence of gas-liquid interfaces makes them highly complicated. Therefore, a number of developments and validations have been conducted on both experimental methods and simulation algorithms to investigate gas-liquid two-phase flows. As for simulation algorithms, interfacial dynamic behaviors are investigated numerically by means of the so-called interface-tracking algorithms. Among them, the volume-of-fluid ${ }^{(1,2)}$, level-set ${ }^{(3)}$ and front-tracking ${ }^{(4)}$ algorithms are widely employed. In addition, the CIP (Cubic-interpolated Pseudo particle) ${ }^{(5)}$ and MPS (Moving Particle Semi-implicit) ${ }^{(6)}$ algorithms are well-known especially in Japan. It is impossible to determine the complete superiority of one algorithm over other algorithms because each

${ }^{*}$ Received 3 Aug., 2009 (No. T1-08-0054) Japanese Original : Trans. Jpn. Soc. Mech. Eng., Vol.74, No.743, B (2008), pp.1512-1521 (Received 22 Jan., 2008) [DOI: 10.1299/jfst.4.711] 
algorithm has advantages and disadvantages.

In our study, a high-precision numerical simulation algorithm has been developed to simulate the vortical-flow-type GE (gas entrainment) phenomenon in a sodium-cooled large-scale fast reactor. In the simulation algorithm, to reproduce the vortical flow behaviors near the system components, e.g. inlet/outlet pipes, an unstructured mesh has been employed to achieve accurate geometrical modeling of such components, and the finite-volume discretizations of the basic equations, i.e. the Navier-Stokes (N-S) and pressure Poisson equations, are conducted. In concrete terms, the first-order Euler explicit, second-order upwind and second-order central schemes are applied to the unsteady, advection and diffusion terms of the N-S equation, respectively. As for the interfacial dynamics, various interface-tracking algorithms were carefully compared, and the volume-of-fluid algorithm was chosen owing to its excellent volume conservation in each phase and its practical performance on applications to various two-phase flow phenomena. In the volume-of-fluid algorithm, the following transport equation of the volume fraction is solved to track interfacial dynamic behaviors:

$$
\frac{\partial f}{\partial t}+\vec{v} \cdot \nabla f=0
$$

where $f$ is the volume fraction of liquid phase with the range from zero to unity. $\vec{v}$ is the velocity vector. In our simulation algorithm, a high-precision volume-of-fluid algorithm, i.e. the PLIC (Piecewise Linear Interface Calculation) algorithm ${ }^{(7)}$, is employed to calculate Eq. (1) accurately. In the PLIC algorithm, first, the unit vector normal to an interface $(\vec{n})$ is calculated in an interfacial cell, and then, the interface is reconstructed as a piecewise linear line. The volume fraction transports through cell-faces are calculated based on the location of the reconstructed interface. The PLIC algorithm and its modifications have been applied to a lot of numerical simulations of various multi-phase flows ${ }^{(8-12)}$, however, they have been developed mainly on structured meshes. In our study, the high-precision numerical simulation algorithm has been developed on unstructured meshes, and therefore, the PLIC algorithm was required to be modified. In previous studies, some proposals were already done for the PLIC algorithms on two-dimensional unstructured meshes ${ }^{(13,14)}$. However, to our knowledge, few studies have been conducted on the extension of the PLIC algorithm to three-dimensional unstructured meshes. Therefore, the authors newly developed the algorithms for the calculation of the unit vector normal to an interface, reconstruction of an interface, volume fraction transport through cell-faces and surface tension with high accuracies and efficiencies on unstructured meshes ${ }^{(15)}$.

In this paper, physics-basis considerations are conducted for mechanical balances at gas-liquid interfaces. By defining momentum and velocity independently at gas-liquid interfaces and developing the momentum transport equations for both gas and liquid phases, the physically appropriate formulation of momentum transport is derived, which eliminated the unphysical behaviors near the gas-liquid interfaces caused by the conventional formulation. In addition, the improvement is also necessary to satisfy the mechanical balances between pressure and surface tension at gas-liquid interfaces and to reduce the spurious velocity at the interfaces, so that the physically appropriate formulation is also derived for the pressure gradient calculation at gas-liquid interfaces. As the validation test, the rising gas bubble in liquid is simulated on the structured and unstructured meshes by the developed simulation algorithms with the physically appropriate formulations.

\section{PLIC Algorithm on Unstructured Meshes}

\subsection{Calculation Algorithm for Unit Vector Normal to Interface}

The unit vector normal to an interface $(\vec{n})$ is calculated based on a given volume 
fraction distribution. In the developed PLIC algorithm, the Gauss-Green theorem ${ }^{(16)}$ is employed to achieve robust and accurate calculation of $\vec{n}$ on unstructured meshes.

\subsection{Algorithm for Reconstruction of Interface}

In the PLIC algorithm, gas-liquid interfaces are reconstructed in each interfacial cell as piecewise linear lines whose direction is normal to the unit vector $\vec{n}$. In addition, the location of the piecewise liner line is obtained so that the partial volume of the interfacial cell determined by the reconstructed interface coincides with the liquid (or gas) volume in the cell. In general, this reconstruction procedure is accomplished by an iterative calculation $^{(8)}$. However, it is reported that a direct (non-iterative) calculation provides more accurate solutions with the reduced computational costs ${ }^{(17)}$ on structured meshes. In our study, the direct calculation algorithm on three-dimensional unstructured meshes was developed based on that algorithm on the two-dimensional unstructured meshes ${ }^{(18)}$.

\subsection{Algorithm for Volume Fraction Transport}

The algorithms for volume fraction transport are roughly classified into two algorithms, i.e. the operator-splitting and multi-dimensional algorithms. The operator-splitting algorithm calculates the volume fraction transports separately in each Cartesian directions while the multi-dimensional algorithm calculates the volume fraction transport in all directions simultaneously. Because it is confirmed that the later is more accurate ${ }^{(9)}$, the multi-dimensional algorithm is chosen in the developed PLIC algorithm.

\subsection{Calculation Algorithm for Surface Tension}

In general volume-of-fluid algorithms, surface tension is calculated in each interfacial cell as body force. In our study, the classical CSF (Continuum Surface Force) algorithm ${ }^{(19)}$ and the RDF (Reconstructed Distance Function) algorithm ${ }^{(20)}$, in which the distance function is determined in each cell based on the locations of reconstructed interfaces, were employed and extended to unstructured meshes.

\section{Physically Appropriate Formulation of Momentum Transport}

In this section, the formulation of momentum transport in the N-S equation is discussed. It must be noted that the following formulations are conducted based on the one-dimensional consideration for simplification, and therefore, the arrows on vector values, e.g. velocity, are omitted in this section.

\subsection{Unphysical Behavior Caused by Conventional Formulation of Momentum Transport}

In conventional finite-volume discretizations, intermediate velocity, which does not satisfy the continuity condition, is calculated in each cell (control volume) by means of the following equations. It must be noted that only the advection term in the N-S equation is discussed here and the diffusion, pressure and external force terms are neglected for simplification.

$$
\begin{aligned}
& m^{n}=\rho_{c}^{n} v^{n}, \\
& F l_{f}=\left(m^{n}+(\nabla m)_{c} d_{c f}\right) v_{f}^{n} \Delta t, \\
& \delta m=\sum_{f} F l_{f}, \\
& m^{n+\delta}=m^{m}+\delta m, \\
& v^{n+\delta}=m^{n+\delta} / \rho_{c}^{n+1},
\end{aligned}
$$


where $m$ is the momentum, $F l$ is the momentum flux and $d_{c f}$ is the vector joining a cell-center to a cell-face-center. The subscripts $c$ and $f$ show the value at a cell-center and on a cell-face, respectively. In Eq. (3), the equation in the curly bracket shows the momentum at a cell-face-center. The summation in Eq. (4) is operated on every cell-face on a cell. The calculation procedure of intermediate velocity is as follows:

1) in a cell, momentum at time level $n\left(\mathrm{~m}^{n}\right)$ is calculated by using velocity and density at time level $n\left(v^{n}\right.$ and $\left.\rho_{c}^{n}\right)$ as Eq. (2);

2) the momentum fluxes $\left(F l_{f}\right)$ through each cell-face during one time increment $(\Delta t)$ are calculated as Eq. (3);

$3)$ by summarizing the momentum fluxes, momentum change in the cell $(\delta m)$ is calculated as Eq. (4);

4) by adding $\delta m$ to $m^{n}$, intermediate momentum $\left(m^{n+\delta}\right)$ is calculated as Eq. (5);

$5)$ intermediate velocity $\left(v^{n+\delta}\right)$ is calculated as $m^{n+\delta}$ divided by density at time level $n+1\left(\rho_{c}^{n+1}\right)$ as Eq. (6).

Though this procedure is appropriate for single-phase simulations, it can be inappropriate for two-phase simulations if large density ratio exists between two phases. For example, one can consider the case that liquid (velocity and density are $v_{l}$ and $\rho_{l}$, respectively) flows into a cell initially filled with gas (velocity and density are $v_{g}$ and $\rho_{g}$, respectively). By considering the continuity condition, it can be assumed that the inlet liquid volume during one time increment $\left(Q_{\text {in }}\right)$ is same as the outlet gas volume during one time increment $\left(Q_{\text {out }}\right)$. Therefore, the intermediate momentum is calculated as

$$
\begin{aligned}
m^{n+\delta} & =m^{n}+\left\{\left(\rho_{l} v_{l}\right) Q_{\text {in }}-\left(\rho_{g} v_{g}\right) Q_{\text {out }}\right\} / V \\
& =\rho_{g} v_{g}+\left(\rho_{l} v_{l}-\rho_{g} v_{g}\right) Q_{i n} / V \\
& =\rho_{g} v_{g}(1-f)+\rho_{l} v_{l} f,
\end{aligned}
$$

where $V$ is the cell volume and $Q_{i n} / V=f$ is used to derive the last equation. Since a time increment $(\Delta t)$ is chosen to be small enough to achieve accurate transports of the volume fraction, $Q_{\text {in }}\left(Q_{\text {out }}\right)$ becomes much smaller than $V$. Therefore, it is natural to assume $f<<1$. In addition, $\rho_{g}<<\rho_{l}$ is imposed (here, $\rho_{l} / \rho_{g}$ is 1,000 by assuming water and air). In this case, the intermediate velocity is calculated as

$$
\begin{aligned}
v^{n+\delta} & =m^{n+\delta} / \rho_{c}^{n+1} \\
& =\left\{\rho_{g}(1-f) v_{g}+\rho_{l} f v_{l}\right\} /\left\{(1-f) \rho_{g}+f \rho_{l}\right\} \\
& =\rho_{g}\left\{(1-f) v_{g}+1000 f v_{l}\right\} /\left\{(1-f) \rho_{g}+f \rho_{l}\right\} \\
& \approx\left(v_{g}+1000 f v_{l}\right) /(1+1000 f) \\
& =\xi v_{g}+(1-\xi) v_{l},
\end{aligned}
$$

where $\xi=1 /(1+1000 f)$. Equation (8) shows that the intermediate velocity is calculated as the mass-weighted average of the gas and liquid velocities. Then, substituting 0.05 and 0.005 as the value of $f$ yields

$$
v^{n+\delta} \approx \begin{cases}0.02 v_{g}+0.98 v_{l} & f=0.05 \\ 0.17 v_{g}+0.83 v_{l} & f=0.005\end{cases}
$$


(a)

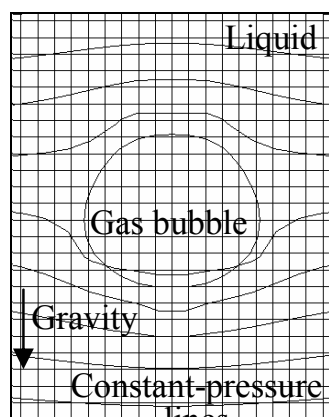

Constant-pressure

Fig. 1 Unphysical pressure distribution caused by inappropriate momentum transport: (a) time level $n$, (b) time level $n+1$

Equation (9) shows that even though the volume fraction is only several percents, the intermediate velocity can be dominated by the liquid velocity. However, this intermediate velocity seems unphysical because the cell is almost occupied by gas phase. In fact, when a rising bubble in liquid is simulated, an unphysical behavior is caused by employing Eqs. (2) to (6). Figure 1 shows the unphysical pressure distribution generated near the lower interface of the rising bubble. By solving the Poisson equation to correct the unphysical intermediate velocities, such an excessive pressure is evaluated near the interface. This unphysical behavior appears only when the interface passes across a cell-face as described above, and the unphysical pressure distribution disappears with the progress of numerical simulations. Therefore, this unphysical behavior is not critical for numerical simulations and seems not to be exposed. However, it is evident that transient simulation results including unphysical behaviors are not reliable.

\subsection{Formulation of Physically Appropriate Momentum Transport}

In this study, physics-basis consideration is conducted to improve the momentum transport calculation. It should be mentioned that the improved formulation was described briefly in our previous work ${ }^{(21)}$, however, the formulation was not useful enough because the momentum variations in gas and liquid phases were not formulated appropriately. In this paper, the further improved formulation is developed with the formulations of the momentum variations. First, gas and liquid phase velocities are independently considered at an interfacial cell, and the velocity and momentum at the interfacial cell are defined as

$$
\begin{aligned}
& v=(1-f) v_{g}+f v_{l}, \\
& m=(1-f) \rho_{g} v_{g}+f \rho_{l} v_{l}
\end{aligned}
$$

It is evident that the velocity calculated by Eq. (10) is the volume-weighted average of the gas and liquid velocities. From Eqs. (10) and (11), the gas and liquid phase velocities at time level $n$ can be calculated as

$$
\begin{aligned}
& v_{g}^{n}=\left(\rho_{l} v^{n}-m^{n}\right) /\left\{\left(1-f^{n}\right)\left(\rho_{l}-\rho_{g}\right)\right\}, \\
& v_{l}^{n}=\left(m^{n}-\rho_{g} v^{n}\right) /\left\{f^{n}\left(\rho_{l}-\rho_{g}\right)\right\} .
\end{aligned}
$$

Then, the improved formulation of the momentum transport is derived with physics-basis considerations. Namely, the momentum transports for gas and liquid phases are conducted independently in the improved formulation as follows: 


$$
\begin{aligned}
& m_{g}^{n+\delta}=\left\{\left(1-f^{n}\right) m_{g}^{n}+\delta m_{g}\right\} /\left(1-f^{n+1}\right), \\
& m_{l}^{n+\delta}=\left(f^{n} m_{l}^{n}+\delta m_{l}\right) / f^{n+1}, \\
& v_{g}^{n+\delta}=m_{g}^{n+\delta} / \rho_{g}, \\
& v_{l}^{n+\delta}=m_{l}^{n+\delta} / \rho_{l} .
\end{aligned}
$$

It should be mentioned that the momentum variations in gas and liquid phases are required to calculate Eqs. (14) and (15). They are calculated as

$$
\begin{aligned}
& F l_{f g}=\left(1-f_{f}\right)\left(m_{g}^{n}+(\nabla m)_{g} d_{c f}\right) v_{f}^{n} \Delta t, \\
& \delta m_{g}=\sum_{f} F l_{f g}, \\
& F l_{f l}=f_{f}\left(m_{l}^{n}+(\nabla m)_{l} d_{c f}\right) v_{f}^{n} \Delta t, \\
& \delta m_{l}=\sum_{f} F l_{f l},
\end{aligned}
$$

where $F l_{f g}$ and $F l_{f l}$ are the momentum fluxes of gas and liquid phases, respectively. $f_{f}$ is the volume fraction on a cell-face. Finally, the intermediate velocity and momentum are calculated by means of Eqs. (10) and (11):

$$
\begin{aligned}
& v^{n+\delta}=\left(1-f^{n+1}\right) v_{g}^{n+\delta}+f^{n+1} v_{l}^{n+\delta}, \\
& m^{n+\delta}=\left(1-f^{n+1}\right) m_{g}^{n+\delta}+f^{n+1} m_{l}^{n+\delta} .
\end{aligned}
$$

The improved formulation can calculate the physically appropriate intermediate velocity at an interfacial cell while the momentum conservation is satisfied completely as well as the conventional equations. This improved calculation procedure is summarized as follows:

1) gas and liquid velocities ( $v_{g}^{n}$ and $\left.v_{l}^{n}\right)$ are calculated by Eqs. (12) and (13);

2) momentum changes are calculated for both the gas and liquid phases $\left(\delta m_{g}\right.$ and $\delta m_{l}$ ) by Eqs. (19) and (21);

3) by adding $\delta m_{g}$ and $\delta m_{l}$ to the gas and liquid momentums at time level $n$ $\left(m_{g}^{n}=\rho_{g} v_{g}^{n}\right.$ and $\left.m_{l}^{n}=\rho_{l} v_{l}^{n}\right)$, intermediate momentums for the gas and liquid phases $\left(m_{g}^{n+\delta}\right.$ and $\left.m_{l}^{n+\delta}\right)$ are calculated by Eqs. (14) and (15);

4) intermediate velocities for the gas and liquid phases $\left(v_{g}^{n+\delta}\right.$ and $\left.v_{l}^{n+\delta}\right)$ are calculated from $m_{g}^{n+\delta}$ and $m_{l}^{n+\delta}$ by Eqs. (16) and (17);

5) intermediate velocity and momentum are calculated by Eqs. (22) and (23).

To validate the improved equations, a rising gas bubble in liquid is simulated again. As a result, the unphysical behavior caused by the conventional formulation is successfully eliminated by the improved formulation (Fig. 2). Therefore, it is concluded that the improved formulation is physically appropriate for numrical simulations of gas-liquid two-phase flows. It should be noted that new variables at interfacial cells need additional memory storage, however, the computational cost does not increase significantly because the number of interfacial cells against whole cells is usually small (only several percents).

\section{Physically Appropriate Formulation of Pressure Gradient}

In this section, a formulation of pressure gradient employed in the N-S and the pressure Poisson equations is discussed. It must be noted that arrows on vector values are omitted also in this section because of one-dimensional considerations. 
(a)

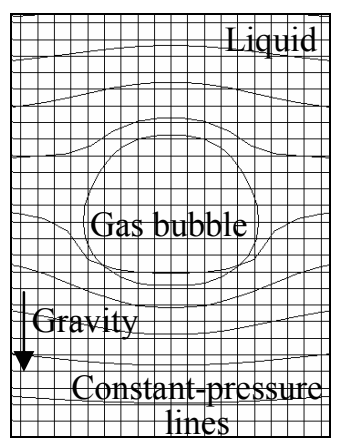

(b)

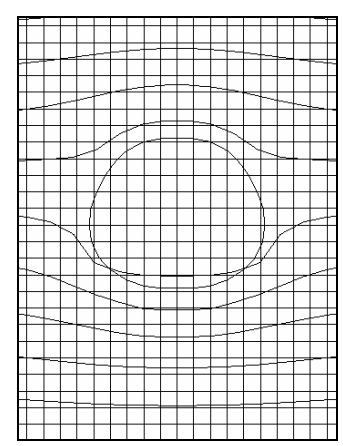

Fig. 2 Physically appropriate momentum transport with improved formulation: (a) time level n, (b) time level $\mathrm{n}+1$

\subsection{Unphysical Behavior Caused by Conventional Formulation of Pressure Gradient}

In our simulation algorithm, the intermediate velocity is obtained by the N-S equation which consists of the pressure and external force terms at time level $n$ in addition to the unsteady, advection and diffusion terms. Then, the intermediate velocity is corrected by the SMAC algorithm ${ }^{(22)}$ to satisfy the continuity condition at time level $n+1$. In the SMAC algorithm, velocity-pressure coupling is achieved by the following equations.

$$
\begin{aligned}
& v^{\prime}=v^{n+\delta}+\delta F_{c} \Delta t / \rho_{c}^{n+1}, \\
& v_{f}^{\prime}=\sum_{\text {sides }} c v^{\prime}, \\
& \nabla \cdot v^{\prime}=\sum_{f} A_{f} \cdot v_{f}^{\prime} / V, \\
& \nabla \cdot\left\{(\nabla \delta p)_{c} / \rho_{c}^{n+1}\right\}=-\nabla \cdot v^{\prime} / \Delta t, \\
& (\nabla \delta p)_{c}=\sum_{\text {adjacent }} \beta \delta p_{c}, \\
& v^{n+1}=v^{\prime}-(\nabla \delta p)_{c} \Delta t / \rho_{c}^{n+1},
\end{aligned}
$$

where the summations in Eqs. (25), (26) and (28) are operated on the cells on both sides of a cell-face, every cell-face on a cell and every cell adjacent to a cell, respectively. $c$ and $\beta$ are the weighting factors for the summations in Eqs. (25) and (28), respectively. They are determined by the spatial relationships between a cell and adjacent cells to the cell. By means of these equations, the velocity at time level $n+1$ is calculated as follows:

1) modified intermediate velocity $\left(v^{\prime}\right)$ is obtained by adding the variation of external force $\delta F_{c}\left(=F_{c}^{n+1}-F_{c}^{n}\right)$ to an intermediate velocity $\left(v^{n+\delta}\right)$ as Eq. (24);

2 ) intermediate cell-face velocity $\left(v_{f}^{\prime}\right)$ is calculated by interpolating $v^{\prime}$ as Eq. (25);

3) velocity divergence $\nabla \cdot v^{\prime}$ at a cell is calculated by summing up the area-weighted $v_{f}^{\prime}$ as Eq. (26);

4) pressure variation $\left(\delta p_{c}\right)$ distribution is determined by solving the pressure Poisson equation to satisfy the continuity condition as Eq. (27);

5) pressure gradient variation $((\nabla \delta p))$ is calculated from $\delta p_{c}$ as Eq. (28);

6) $v^{\prime}$ is updated to velocity at time level $n+1\left(v^{n+1}\right)$ by using $(\nabla \delta p)_{c}$ as Eq. (29).

The above formulation is valid for numerical simulations of gas-liquid two-phase flows where only uniform external force (e.g. gravitational force) works. However, an unphysical behavior can be caused by the unbalance between pressure gradient and surface tension at interfacial cells. In fact, when a rising gas bubble in liquid is simulated, discontinuous 
velocity distribution appears near a gas-liquid interface as shown in Fig. 3(a). In the above equations, Eqs. (24) to (27) are physically appropriate because the force balance between pressure gradient and surface tension is satisfied on every cell-face, that is, the physically appropriate pressure distribution is achieved. Therefore, it is evident that the force unbalance is originated in the calculation of pressure gradient (Eq. (28)). In concrete terms, to guarantee a physically appropriate force balance between pressure gradient and surface tension at an interfacial cell, the pressure gradient in the interfacial cell should be improved.

\subsection{Formulation of Physically Appropriate Pressure Gradient}

In order to improve Eq. (28), first, the pressure variation on a cell-face $\left(\delta p_{f}\right)$ is introduced. Then $\delta p_{f}$ is defined to satisfy the physically appropriate force balance between pressure gradient and external force at an interfacial cell. Finally, pressure gradient variation is calculated by using $\delta p_{f}$. In this procedure, $\delta p_{f}$ is defined as

$$
\begin{aligned}
& \delta p_{f}=\delta p_{c}+(\nabla \delta p)_{c}^{\prime} d_{c f} / 2, \\
& \left\{\delta F_{c}-(\nabla \delta p)_{c}^{\prime}\right\} / \rho_{c}^{n+1}=\sum_{\text {sides }} c \delta F_{c} / \rho_{f}^{n+1}-(\nabla \delta p)_{f} / \rho_{f}^{n+1},
\end{aligned}
$$

where $(\nabla \delta p)_{c}^{\prime}$ is the temporal value of pressure gradient variation for calculating $\delta p_{f}$. The following equation for calculating the pressure gradient variation is introduced instead of Eq. (28):

$$
\nabla \delta p=\sum_{f} A_{f} \delta p_{f} / V
$$

Equations (30) and (32) represent that $\delta p_{f}$ can be calculated based on the external force variation when the force balance between the variations of pressure gradient and external force is satisfied on the cell-face. Therefore, in Eq. (32), the pressure gradient variation is calculated at an interfacial cell by considering the force balance between the variations of the pressure gradient and external force on every cell-face on the interfacial cell. To validate the improved formulation (Eqs. (30), (31) and (32)), a rising gas bubble in liquid is simulated again. Figure 3(b) shows the simulation result of velocity distribution around the rising gas bubble. The discontinuous velocity distribution caused by the conventional formulation (shown in Fig. 3(a)) is completely eliminated. This result shows that the improved formulation of pressure gradient can lead physically appropriate solutions for numerical simulations of gas-liquid two-phase flows with surface tension.

It should be noted that the above discussion is conducted based on the SMAC algorithm, however, the same discussion is established for other velocity-pressure coupling algorithms, e.g. the SIMPLE (Semi-Implicit Method for Pressure Linked Equation) $\operatorname{algorithm~}^{(23)}$.

\section{Validation of Developed Simulation Algorithms}

As the validation of the simulation algorithms developed in this paper, a simple problem on gas-liquid two-phase flow is addressed in this section.

\subsection{Simulation Conditions for Rising Bubble in Liquid}

The transient behavior of a rising gas bubble in liquid is simulated to validate the applicability of the developed high-precision numerical simulation algorithm to gas-liquid two-phase flows. It is well known that transient behaviors of rising gas bubbles in liquid are categorized by the Morton $(M o)$ and Eötvös (Ë̈) numbers: 
(a)

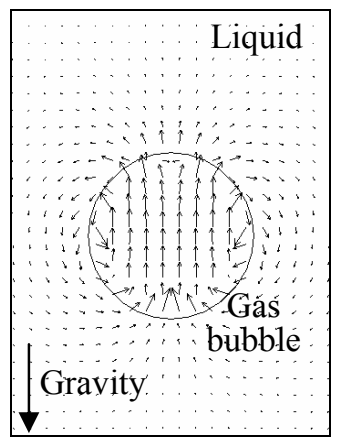

(b)

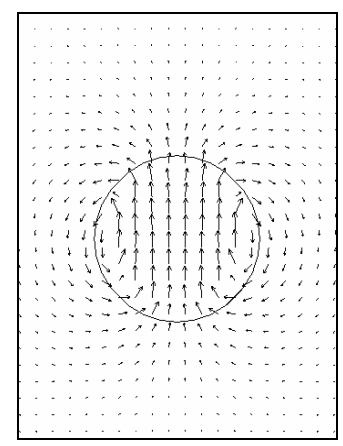

Fig. 3 Velocity distribution near gas bubble interface: (a) unphysical distribution, (b) physically appropriate distribution by improved formulation

$$
\begin{aligned}
& M o=|g| \mu_{l}^{4} / \rho_{l} \sigma^{3}, \\
& E \ddot{O}=|g| d_{e}^{2} \rho_{l} / \sigma,
\end{aligned}
$$

where $\mu_{l}$ is the viscosity of liquid phase, $d_{e}$ is the volume-equivalent diameter of a gas bubble. For example, a number of experiments with different couplings of the Morton and Eötvös numbers have been performed and summarized ${ }^{(24)}$. In this study, the numerical simulations are performed on two-dimensional Cartesian meshes under the simulation conditions used in the previous similar study ${ }^{(25)}$. As shown in Table 1, the Morton number is fixed to be the value of water at room temperature, and the Eötvös number is changed as the simulation parameter. The bubble shape in the experiments becomes Spherical Cap, Spherical Cap Ellipsoidal, Ellipsoidal and Spherical under each Eötvös number condition.

Table 1 Simulation conditions

\begin{tabular}{cccl}
\hline Case & Mo & $E \ddot{o}$ & \multicolumn{1}{c}{ Bubble shape in experiment } \\
\hline 1 & $2.58 \times 10^{-11}$ & $5.32 \times 10^{1}$ & Spherical cap \\
2 & & $1.73 \times 10^{1}$ & Spherical cap $\sim$ Ellipsoidal \\
3 & & $3.32 \times 10^{0}$ & Ellipsoidal \\
4 & $1.80 \times 10^{-1}$ & Spherical
\end{tabular}

Figure 4 shows the simulation domain and the simulation meshes. The simulation domain is a rectangular region with the height and width of $160 \mathrm{~mm}$ and $80 \mathrm{~mm}$, respectively. This domain is subdivided by uniform square cells $(3,200$ cells) with the size of $2.0 \times 2.0 \mathrm{~mm}$ i in the structured mesh (Fig. 4(a)), and is subdivided into 3,234 triangular cells in the unstructured mesh (Fig. 4(b)). In the unstructured mesh, a highly-distorted unstructured mesh is generated intentionally to validate the simulation ability on low-quality meshes. The center of the circular gas bubble with a radius of $20 \mathrm{~mm}$ is located initially at a height of $30 \mathrm{~mm}$ from the bottom of the domain. Initial velocity and pressure are zero in all locations. The transient behaviors of the air bubble are investigated for 0.3 second under each simulation condition. The non-slip conditions are applied to the bottom and side walls of the domain, and the constant pressure condition $(p=0)$ is applied to the upper boundary.

\subsection{Simulation Results of Rising Bubble in Liquid}

Figures 5, 6, 7 and 8 show the simulation results of the transient rising bubble behaviors on the structured mesh (bubble shapes and velocity distributions around the bubble). In each figure, the rising bubbles gradually change their shapes with time and reach the terminal shapes. Physically, the smaller Eötvös number means the stronger surface tension effect which works to keep the initial circular bubble shape. It is obvious that this tendency is 
reproduced in the simulation results. In addition, the terminal gas bubble shapes in the simulation results correspond well with the experimental data under each Eötvös number. Figure 9 shows the simulation result on the unstructured mesh under $E \ddot{o}=3.32 \times 10^{0}$. It is evident that the simulation result on the unstructured mesh agrees well with the corresponding simulation result on the structured mesh (Fig. 7) at each elapsed time. Especially, the gas bubble shapes at each elapsed time are almost same in both simulation results. Therefore, it is concluded that the high-precision numerical simulation algorithm developed in this study is able to simulate gas-liquid two-phase flows accurately even on unstructured meshes.

(a)

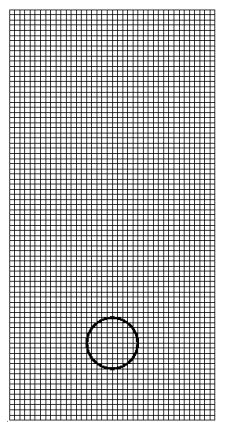

(b)

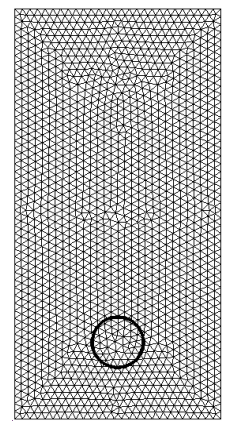

Fig. 4 Simulation meshes: (a) structured mesh, (b) unstructured mesh

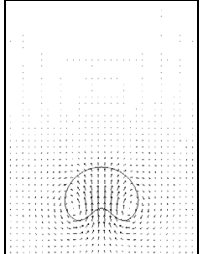

$t=0.05 \mathrm{~s}$

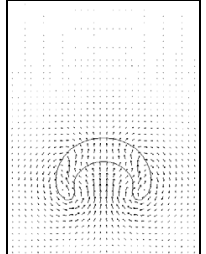

$t=0.10 \mathrm{~s}$

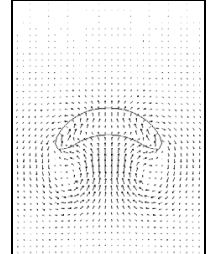

$t=0.15 \mathrm{~s}$

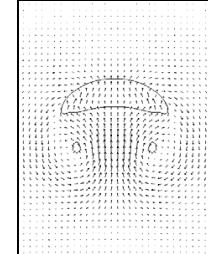

$t=0.20 \mathrm{~s}$

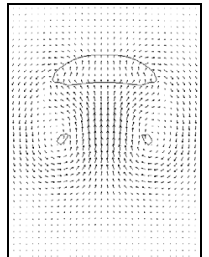

$t=0.25 \mathrm{~s}$

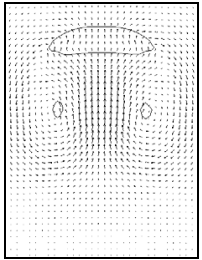

$t=0.30 \mathrm{~s}$

Fig. 5 Transient behavior of gas bubble (structured mesh, $E \ddot{o}=5.32 \times 10^{1}$ )

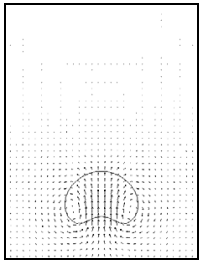

$t=0.05 \mathrm{~s}$

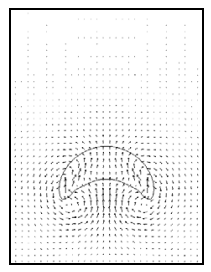

$t=0.10 \mathrm{~s}$

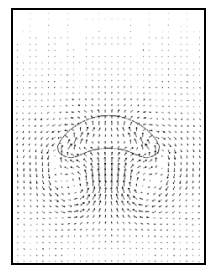

$t=0.15 \mathrm{~s}$

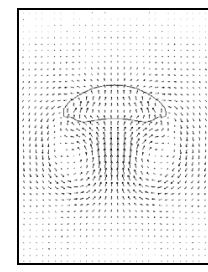

$t=0.20 \mathrm{~s}$

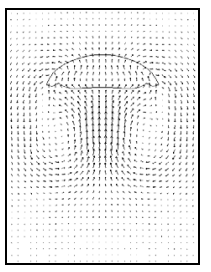

$t=0.25 \mathrm{~s}$

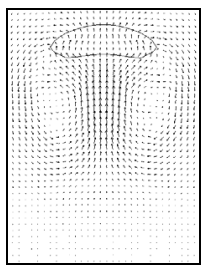

$t=0.30 \mathrm{~s}$

Fig. 6 Transient behavior of gas bubble (structured mesh, $E \ddot{o}=1.73 \times 10^{1}$ )

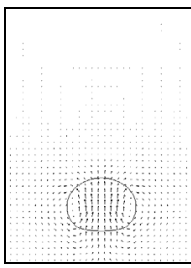

$t=0.05 \mathrm{~s}$

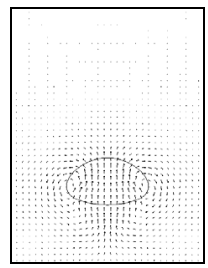

$t=0.10 \mathrm{~s}$

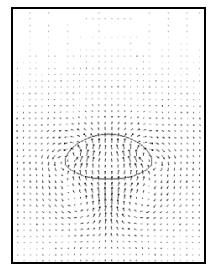

$t=0.15 \mathrm{~s}$

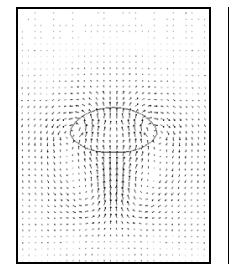

$t=0.20 \mathrm{~s}$

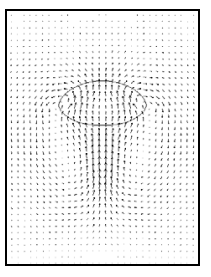

$t=0.25 \mathrm{~s}$

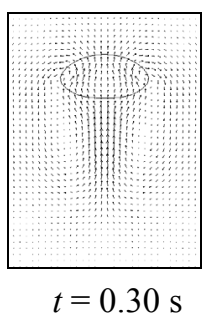

$t=0.30 \mathrm{~s}$

Fig. 7 Transient behavior of gas bubble (structured mesh, $E \ddot{O}=3.32 \times 10^{0}$ ) 


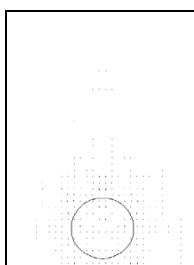

$t=0.05 \mathrm{~s}$

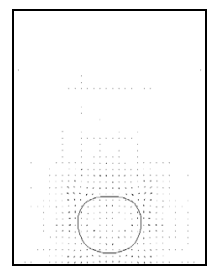

$t=0.15 \mathrm{~s}$

$t=0.20 \mathrm{~s}$

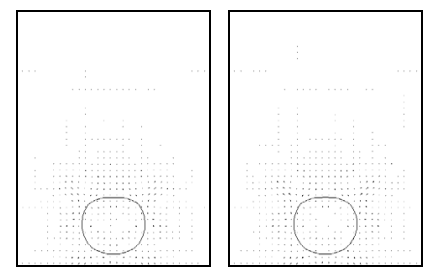

$t=0.25 \mathrm{~s}$

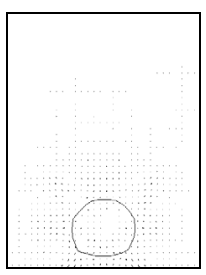

$t=0.30 \mathrm{~s}$

Fig. 8 Transient behavior of gas bubble (structured mesh, $E \ddot{o}=1.80 \times 10^{-1}$ )

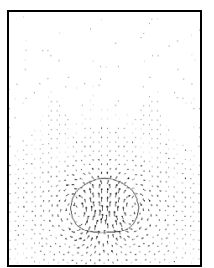

$t=0.05 \mathrm{~s}$

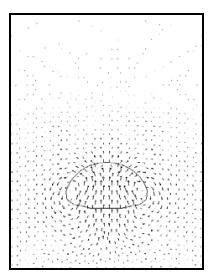

$t=0.10 \mathrm{~s}$

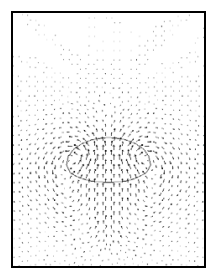

$t=0.15 \mathrm{~s}$

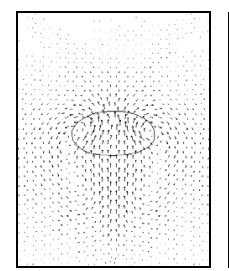

$t=0.20 \mathrm{~s}$

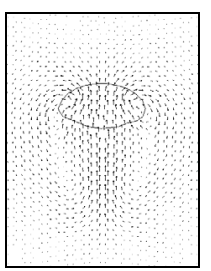

$t=0.25 \mathrm{~s}$

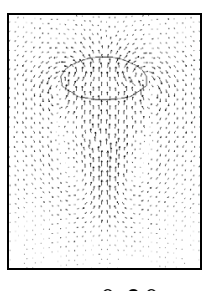

$t=0.30 \mathrm{~s}$

Fig. 9 Transient behavior of gas bubble (unstructured mesh, $E \ddot{o}=3.32 \times 10^{0}$ )

\section{Conclusion}

As a part of the development of the high-precision numerical simulation algorithm for gas-liquid two-phase flows on unstructured meshes, the formulations of momentum transport and pressure gradient at the interface are improved based on physics-basis considerations for mechanical balances at a gas-liquid interface. First, the unphysical behaviors near the gas-liquid interface of a rising bubble in liquid caused by the conventional formulations were pointed out. Then, by defining momentum and velocity independently at gas-liquid interfaces and developing the momentum transport equations for both gas and liquid phases, the physically appropriate formulation of momentum transport was derived, and the unphysical pressure distribution was eliminated successfully. In addition, the improvement is also achieved to satisfy the mechanically appropriate balances between pressure and surface tension at gas-liquid interfaces. As a result, the improved formulation of the pressure gradient calculation at the interface was efficient to reduce the spurious velocity near the interface. To validate the developed high-precision numerical simulation algorithm with the physically appropriate formulations, the numerical simulation of a rising gas bubble in liquid was performed. As a result, the simulated terminal bubble shapes on the structured and highly-distorted unstructured meshes coincided with the experimental data under each simulation condition determined by the Morton and Eötvös numbers. Therefore, it was confirmed that the high-precision numerical simulation algorithms provide accurate simulation results of interfacial dynamic behaviors.

\section{References}

(1) Hirt, C. W. and Nichols, D. B., Volume of Fluid (VOF) Method for the Dynamics of Free Boundaries, J. Comput. Phys., Vol. 39 (1981) pp. 201-205.

(2) Noh, W. F. and Woodward, P., SLIC (Simple Line Interface Calculation), Lecture Notes in Physics (van der Vooren, A. I. and Zandbergen, P. J. eds.), Springer-Verlag (1976) pp. 330-340.

(3) Osher, S. and Sethian, J. A., Fronts Propagating with Curvature-Dependent Speed: Algorithms based on Hamilton-Jacobi Formulations, J. Comput. Phys., Vol. 79 (1988) pp. 12-49.

(4) Unverdi, S. O. and Tryggvason, G., A Front-Tracking Method for Viscous, Incompressible 
Multi-Fluid Flows, J. Comput. Phys., Vol. 100 (1992) pp. 25-37.

(5) Takewaki, H. and Yabe, T., The Cubic-Interpolated Pseudo Particle (CIP) Method: Application to Nonlinear and Multi-Dimensional Hyperbolic Equations, J. Comput. Pyhs., Vol. 70 (1987) pp. 355-372.

(6) Koshizuka, S. and Oka, Y., Moving-Particle Semi-Implicit Method for Fragmentation of Incompressible Fluid, Nucl. Sci. Eng., Vol. 123 (1996) pp. 421-434.

(7) Youngs, D. L., Time-Dependent Multi-Material Flow with Large Fluid Distortion, Numerical Methods for Fluid Dynamics (Morton, K. W. and Baines, M. J. eds.), Americal Press, New York (1982) pp. 273-486.

(8) Rider, W. and Kothe, D. B., Reconstructing Volume Tracking, J. Comput. Phys., Vol. 141 (1998) pp. 112-152.

(9) Pilliod, J. E. and Puckett, E. G., Second-Order Accurate Volume-of-Fluid Algorithms for Tracking Material Interfaces, J. Comput. Phys., Vol. 199 (2004) pp. 465-502.

(10) Harvie, D. J. E. and Fletcher, D. F., A New Volume of Fluid Advection Algorithm: the Stream Scheme, J. Comp. Phys., Vol. 162 (2000) pp. 1-32.

(11) Kunugi, T., MARS for Multiphase Calculation, Computational Fluid Dynamics J., Vol. 19 (2001) pp. 563-571.

(12) Lopez, J., Hernandez, J., Gomez, P. and Faura, F., An Improved PLIC-VOF Method for Tracking Thin Fluid Structures in Incompressible Two-Phase Flows, J. Comput. Phys., Vol. 208 (2005) pp. 51-74.

(13) Ashgriz, N., Barbat, T. and Wang, G., A Computational Lagrangian-Eulerian Advection Remap for Free Surface Flows, Int. J. Numer. Meth. Fluids, Vol. 44 (2004) pp. 1-32.

(14) Shahbazi, K., Paraschivoiu, M. and Mostaghimi, J., Second Order Accurate Volume Tracking based on Remapping for Triangular Meshes, J. Comput. Phys., Vol. 188 (2003) pp. 100-122.

(15) Ito, K., Yamamoto, Y. and Kunugi, T., Development of Numerical Method for Simulation of Gas Entrainment Phenomena, Proc. The Twelfth International Topical Meeting on Nuclear Reactor Thermal Hydraulics, Pittsburgh, PA, Sep. 30 - Oct. 5 (2007).

(16) Kim, S. E., Makarov, B. and Caraeni, D., A Multi-Dimensional Linear Reconstruction Scheme for Arbitrary Unstructured Grids, Proc. 16th AIAA Computational Fluid Dynamics Conference, Orlando, FL, June 23-26 (2003).

(17) Scardvelli, R. and Zaleski, S., Analytical Relations Connecting Linear Interface and Volume Functions in Rectangular Grids. J. Comp. Phys., Vol. 164 (2000) pp. 228-237.

(18) Yang, X. and James, A. J., Analytic Relations for Reconstructing Piecewise Linear Interfaces in Triangular and Tetrahedral Grids, J. Comp. Phys., Vol. 214 (2006) pp. 41-54.

(19) Brackbill, J. U. D., Kothe, B. and Zemach, C., A Continuum Method for Modeling Surface Tension, J. Comput. Phys., Vol. 100 (1992) pp. 335-354.

(20) Cummins, S. J., Francois M. M. and Kothe, D. B., Estimating Curvature from Volume Fractions. Computer \& Structure, Vol. 83 (2005) pp. 425-434.

(21) Ito, K., Kunugi, T., Ohshima, H. and Kawamura, T., Formulations and Validations of a High-precision Volume-of-fluid Algorithm on Non-orthogonal Meshes for Numerical Simulations of Gas Entrainment Phenomena, J. Nucl. Sci. Tech., Vol. 46 (2009) pp. 366-373.

(22) Amsden, A. A. and Harlow, F. H.. The SMAC Method: a Numerical Technique for Calculating Incompressible Fluid Flows, Technical Report, Los Alamos National Laboratory (1970) LA-4370.

(23) Patankar, S. V., Numerical Heat Transfer and Fluid Flow, McGraw-Hill, New York (1980).

(24) Grace, J. R., Shapes and Velocities of Bubbles Rising in Infinite Liquid, Trans. Instn Chem. Engrs., Vol. 51 (1973) pp. 116-120.

(25) Yoon, H. Y., Koshizuka, S. and Oka, Y., Direct Calculation of Bubble Growth, Departure, and Rise in Nucleate Pool Boiling, Int. J. Multiphase Flow, Vol. 27 (2001) pp. 277-298. 